\title{
7. CELESTIAL MECHANICS (MÉCANIQUE CÉLESTE)
}

PRESIDENT: P. J. Message.

VICE-PRESIDENT: V. Szebehely.

ORGANISING COMmITTEE: E. P. Aksenov, V. A. Brumberg, M. S. Davis, A. Deprit, G. N. Duboshin, G. E. O. Giacaglia, G. Hori, Y. Kozai, B. Morando, L. Sehnal.

This report is written both to indicate the topics within the main field of Celestial Mechanics on which research has been carried out in the last three years, as well as to assist the enquirer who seeks the published work on a particular topic. To approach both of these objectives within the requirement of brevity, it has seemed best to collect together, according to topic, most (though inevitably, alas, not all) of the work in the Commission's field published in the period 1973 to 1975 . I am very grateful to Dr V. K. Abalakin for a most helpful review of research in the U.S.S.R., and to all colleagues who sent me details of their work. Each paper is indicated, where possible, by a reference in the form (v.s.a.), where a mention of the paper (with a full reference to the original journal of publication) is numbered ' $a$ ', in section 's', of volume ' $\mathrm{v}$ ' of Astronomy and Astrophysics Abstracts. Where no such mention has appeared at the time of writing, the reference given is to the journal of publication, followed by the volume number, the page reference, and the year of publication. The following abbreviations are used, as well as the standard ones:

$\begin{array}{llll}A A= & \text { Astron. Astrophys. } & C M= & \text { Celes. Mech. } \\ A J= & \text { Astron. J. } & C R= & \text { Compt. Rend. Acad. Sci. Paris } \\ A N= & \text { Astron. Nachr. } & M N= & \text { Monthly Notices Roy. Astron. Soc. } \\ A Z= & \text { Astron. Zh. (Akad. Nauk SSSR) } & T K A O= & \text { Trudy Kazan Gorod Astron. Obs. } \\ B I T A= & \text { Bjull. Inst. Teor. Astron. } & & \end{array}$

Beginning with general analytical theory in celestial mechanics, there has been much fruitful work on transformations of the Lie series and Von Zeipel types, their extensions, and equivalences between the various formulations, including that of J.S. Choi and B. Tapley (09.042.003), J. S. Griffith (09.042.038), Y. Thiry (Comm. 92e Congrès de l'Assoc. pour l'Avancement des Sci., 1973), U. Kirchgraber (09.042.044 and 10.042.094), the latter with M. Vitins (CM 12, 139, 1975), L. M. Berkovitch and B. E. Gel'fat (09.042.048), R. Cid and M. Calvo (10.042.001), the latter with J. M. Correas ( $C M$ 12, 131, 1975), J. Henrard (10.042.081), the latter with J. Roels (12.042.077), M. Rapaport (11.042.016), K.R. Meyer (11.042.064), N. Watanabe (12.042.013), J. Moser (12.042.028), K. V. Kholshevnikov (12.042.065), and J. Meffroy ( $C R 280 \mathrm{~A}, 21,1975)$. Much of this work has also been concerned with the stability of particular solutions in Hamiltonian systems, as has that of J. Hadjidemetriou (10.042.095), N. Burgoyne and R. Cushman (11.042.008), R. McGehee (11.042.049), M.A. Vashkov'yak (12.042.069), F. I. Kiselov (12.042.080), and G. Merman (BITA 14, 37, 1975). Also on this topic, with especial reference to resonance with the variational frequencies, and periodic solutions associated with them, is the work of K. T. Alfriend and D. L. Richardson (09.042.040), D. S. Schmidt (11.042.013), the latter with D. Sweet (J. Diff. Equations 14, 597, 1973), and G. I. Shir$\min (12.042 .016)$. Further advances in the ideal resonance problem and its extensions have been made by B. Garfinkel (09.042.009, 10.042.021, CM 12, 208, 1975), the latter with C.A. Williams (11.042.014), and by A. H. Jupp (09.042.004, with errata in .901, 09.042.019, $10.042 .022,11.042 .011,12.042 .039)$. The latter writer has applied his solution to the free rotation of a rigid body (11.042.012). L. Losco (10.042.010 and 12.042.041) has studied an integral invariant deriving from Poincaré and Cartan. G. Aymerich (09.062.035) studied integral invariants, and F. Nahon (10.021.014) particular solutions of Levi-Civita. C. Froeschlé 
(10.042.030), and the latter with J.P. Scheidecker (12.151.017), and F. Rannou (11.042.021) have made numerical studies of mappings and dynamical systems, in the study of the phenomenon of 'wildness'. Further developments in the study of the regularisation of the Kepler problem, and applications to perturbation theory, are due to P. E. Kustaanheimo (11.052.028, 12.042.055, Phys. Fennica 8, 7, 1973, Soc. Sci. Fennica Comm. Phys. Math. 44, 1, 1974, CR $279 \mathrm{~A}, 675,1974$, and $A N$ 296, 163, 1975), E. Stiefel (10.042.065), U. Kirchgraber (10.042.026), O. Volk (10.042.032), F. A. E. Pirani (11.042.085), V. R. Bond (12.042.058), and $\mathrm{R}$. Broucke and $\mathrm{H}$. Lass ( $C M 12,317,1975)$. Regularisation of the two-body problem with variable masses was studied by P. Guillaume (12.042.015), and J. P. Vinti (12.042.062) has given a solution of the two-body problem with ' $G$ ' varying inversely to a linear function of time. Integrable cases of the three-body problem with variable masses have been given by $B$. $E$. Gel'fat (12.042.083), and the perturbed Kepler problem with variable masses has been studied by T. B. Omarov (10.042.040) and F. Verhulst (CM 11, 95, 1975). J. P. Vinti (10.042.025) has given a quadrature solution for general relativistic motion, and the relation between time scales has been studied by V. S. Nuovio $(12.006 .060)$ and J. B. Thomas $(A J 80,405,1975)$. New forms of the Gaussian perturbation equations have been given by J. P. Vinti (09.042.020) to non-Keplarian reference orbits, by S. N. Yashkin (11.042.071), M. Silver (CM 11, 39, 1975), and R. Sridharan and M. L. Renard (CM 11, 179, 1975), and by G. H. Born, E. J. Christensen and L. K. Seversike (11.052.003), using a new form of universal variable. Trajectory deviations and optimal orbits have been studied by E. L. Liipola (11.042.060) and D. J. Jezewski $(C M$ 12, 297, 1975).

Turning to work relating to the analytical development of perturbation theories, methods of expansion of co-ordinates and velocity components have been studied by $E$. Fichera (11.042.063), K. Ahmad (11.042.072), R. Broucke (12.042.075), the latter with P. Cefola (09.042.022), and, for hyperbolic motion, by A. Kahkorov (12.042.085). New work on the expansion of the disturbing function has been done by C. A. Altavista (09.042.025), E. I. Timoshkova (09.042.030), H. Kinoshita, G. Hori and H. Nakai (11.042.053), V. B. Titov (11.042.069), N. Abu-el Ata and J. Chapront $(A A 38,57,1975)$, E. Z. Khotimskaya (BITA 14, $47,1975)$, and, using ellipsoidal co-ordinates, E. J. Burstein ( $C M 11,255,1975)$. The calculation of high-order perturbations is the subject of studies by Yu. V. Plakhov (09.042.014, and $.036,11.042 .044$, and .075). Questions arising in the determination of orbits underly the work of E. E. Burniston and C. E. Siewert (09.042.010 and 12.042.004), K. V. Kholshevnikov (09.042.056), and C. Simo (12.042.073). Methods for computer handling of multiple Fourier series, including algebraic manipulation, have been developed by J. R. Cherninck (09.042.005), J. Chapront, M. Chapront, and J. L. Simon (11.042.020), S. G. Kapralov (11.042.076), R. J. Fateman (12.042.021), A. L. Kutuzov (12.042.086), L. A. Moskovkina (12.042.087), V. A. Brumberg and L. A. Isakovich (12.042.051, and Algorithms of Celestial Mechanics, Inst. Teor. Astron., Leningrad, No. 4, 75), and P. J. Shelus and W. H. Jefferys (CM 11, 75, 1975). Increased efficiency in the storage of ephemerides is achieved in a method employing Chebyshev polynomials, devised by A. Deprit, W. Poplarchek, and A. Deprit-Bartholomé (CM 11, 53, 1975). J. D. Mulholland (09.042.021) has studied the avoidance of badly-determined equations in differential correction.

Considering methods of numerical integration, of especial utility in celestial mechanics, D. G. Bettis (10.021.003), and E. Everhart (12.042.006) have studied developments of the Runge-Kutta type, while A. E. Roy, W. Black and P. E. Moran (09.042.006 and .011, 10.042.059 and .061) have developed a Taylor series method based on recurrence relations for the $n$-body problem, and 0 . Graf $(11.042 .036)$ has studied multi-revolution methods. V. F. Lager and A. T. Onishchenko (12.042.070) have given two multi-step analy tical methods. Refinement of conventional methods has been undertaken by M. S. Yarov-Yarovoy (Trudy Sternberg Astron. Inst. 45, 179, 1974). J. Baumgarte (10.042.024), and the latter with E. Stiefel (11.042.034 and 12.042.007), N. Sigrist (Z. Angew. Math. Phys., 1974), C. E. Velez (12.042.074), and K. Zare and V. Szebehely (CM 11, 469, 1975) have studied methods of stabilisation by means of transformation of the equations of motion.

Reviews of recent work on planetary and satellite motion have been made by G. A. Wilkins and A. T. Sinclair (11.091.028), by V. A. Brumberg and G. A. Krasinsky (Usp. Fiz. Nauk 114, 
377, 1974), and by J. M. A. Danby (11.042.037). Quasi-periodic motions for the major planets of the solar system have been computed by V. A. Brumberg, L. S. Evolokunova and V. I. Skripnichenko ( $C M 11,131,1975$ and $A Z$ 52, 420, 1975), and the resonances between three or more mean motions, of zero order in the eccentricities and inclinations, identified. J. Meffroy and O. S. M. Kamel (12.042.014 and .054) have worked to second order in the eccentricities and inclinations, using Poincaré's variables and Von Zeipel's method, examining the critical terms arising from the near-commensurabilities of orbital period between Jupiter and Saturn, and between Uranus and Neptune. P. Bretagnon (11.042.001) has worked to fourth order in the eccentricities and inclinations. A new set of variables for the calculation of secular perturbations in the case of close commensurability has been given by N. I. Lobkova and M. S. Petrovskaya (12.042.037). C. J. Cohen, E. C. Hubbard and C. Oesterwinter $(09.091 .060$ and 11.042.066), using results of their numerical integrations, have given the variations of the orbital elements of the major planets over a period of ten million years. J. Birn (09.091.017), using numerical integration experiments with fictitious planetary systems in which the planetary masses are augmented to increase the impact of the perturbations, finds zones both of stability and instability in the ratios of the major semi-axes. Note that, however, in the formal asymptotic series for the planetary elements, the expressions for the major semi-axes differ from constants only by periodic terms, to all orders in the perturbations, when periods up to those arising in the classical secular variation theory are taken into account (see P. J. Message, Proc. Adv. Study Inst. on Long Term Prediction in Dynamics, Cortina, 1975). V. A. Brumberg (12.042.036), J. Chapront ( $C M 11,379,1975)$, and T. V. Ivanova $(A Z 52,839,1975)$ have developed iterative methods for the construction of general planetary theories. V. A. Brumberg and J. Chapront (10.042.058) have constructed a general theory to first order, identifying the resonant terms. J. L. Simon and J. Chapront (11.042.042) have derived second-order perturbations for Jupiter and Saturn, and carried out comparison with Leverrier. J. L. Simon and P. Bretagnon $(A A 42,259,1975)$ have constructed first-order perturbations for the four great planets, using Poincaré variables. J. Meffroy $(10.042 .089)$ has used the Lie series method to develop the short-period terms to second order, using Henrard's variables, related to those of Poincaré. P. E. Nacozy and R. E. Diehl (11.101.001) have investigated the libration phenomena underlying the long-period perturbations of Pluto by Neptune, concluding that Pluto's apse regresses on average relative to Neptune's. A review of values for the masses of the planets and natural satellites has been made by R. L. Duncombe, W. J. Klepczynski and P. E. Seidelmann (Fundam. Cosmic Phys. 1, 119, 1973), and a set of mean elements is given by P. K. Seidelmann, L. E. Doggett, and M. R. de Luccia (11.042.022). Elements of Mars' orbit have been given by N. I. Glebova (BITA 3,612, 1975). Mass and position limits for a possible tenth major planet have been given by D. Rawlins and M. Hammerton (09.101.016).

Developments in analytical lunar theory have been made by A. Bec, J. Kovalevsky and C. Meyer (10.094.103), M. Chapront-Tourie (12.042.063), G. F. Osipov (09.094.116), and a suggestion for the use of the Saros has been made by A. E. Roy (09.094.042). Corrections to the constants of the orbit and limb charts have been derived from Washington observations from 1925 to 1968 by L. N. Kizyun (09.094.919). G. I. Eroshkin (12.094.005) has studied the effect of the Moon's figure on its motion, through the resonance between its rotational and orbital periods. L. I. Rumyantseva $(12.094 .001)$ has carried out numerical integration in lunar theory, improving the orbital data. The theory of the physical libration has been studied by K. S. Shakirov (11.094.007, and Astron. Circ. 853, 5, 1975) and an extended analy tical theory is being developed by A. H. Cook. Our knowledge of the Moon's motion and physical libration are being very greatly increased by the impact of laser ranging data; we note the work of V.A. Abalakin, O. Calame, Yu. L. Kokurin, J. D. Mulholland, A. Orszay, and E. C. Silverberg (09.094.166), P. L. Bender, D. G. Currie, R. H. Dicke, D. H. Eckhardt, J. E. Faller, W. M. Kaula, J. D. Mulholland, H. H. Plotkin, S. K. Poultney, E. C. Silverberg, D. T. Wilkinson and C. O. Alley (10.094.074), W. M. Kaula and P. A. Baxa (10.094.085), J. G. Williams, M. A. Slade, D. H. Eckhardt and W. M. Kaula (10.094.107), J. D. Mulholland and P. J. Shelus (10.094.111), R. I. Abbott, P. J. Shelus, J. D. Mulholland, and E. C. Silverberg (10.094.101), the last three authors $(A J 80,154,1975)$, and V. K. Abalakin, N. N. Boyko, Yu. L. Kukurin and V. F. Lobanov $(A Z 52,387,1975)$. The lunar acceleration, secular changes in the Earth's rotation, and 
possible changes in the Newtonian gravitational factor ' $G$ ' have been investigated by $L$. V. Morrison (09.044.003), R. R. Newton (11.044.032), and T. C. van Flandern (MN 170, 333, 1975).

The motions of Mars' satellites were studied by V. A. Shor (12.097.205, and $C M 12,61$, 1975), and epemerides from Mariner 9 data have been given by G. H. Born and T. C. Duxbury (CM 12, 77, 1975). The development of new analytical theories of the Galilean satellites of Jupiter have been in hand by S. Ferraz-Mello (12.099.228 and $C M 12,27,1975)$, and by J. L. Sagnier $(09.099 .032$ and $C M 12,19,1975)$, and a new treatment of the commensurabilities and librations has been given by A. T. Sinclair $(C M 12,89,1975$, and $M N 171,59$, 1975). A method of revitalising Sampson's theory has been proposed by J. H. Lieske (11.099.206, and $C M 12,5,1975)$, who also studied the 3:7 near-commensurability of periods between Ganymede and Callisto (10.099.005). Work on the observations of the positions and mutual phenomena of the Galilean satellites, and comparison with theory, has been done by K. Aksnes (11.099.201), J. E. Arlot ( $C M$ 12, 39, 1975), H. Davila, S. Debardat and A. Journet (CM 12, 51, 1975), T. C. Duxbury, T. V. Johnson and D. L. Matson (Icarus 25, 569, 1975), S. J. Goldstein (09.099.023, and $A J$ 80, 532, 1975), and C. F. Peters $(10.099 .066$, and $C M 12$, 99, 1975). The motion of Jupiter's satellite Amalthea (V) has been studied by V. A. Tamarov (10.099.087), and V. G. Demin (Astron. Circ. 2, 1973), that of the sixth satellite by L. E. Bykova (10.099.088 and .090), the seventh by T. V. Bordovitsyna (09.099.102, 10.099.062 and .089), and the sixth, ninth, and tenth by L. D. Andreyeva and L. E. Bykova (12.099.100). Ephemerides of Jupiter's satellites VIII to XII have been given by K. A. Aksnes (09.099.022), and for satellite XIII by C. T. Kowal, K. Aksnes, B. G. Marsden and E. Roemer $(A J 80,460,1975)$. A theoretical study of the motions of Saturn's satellites Enceladus and Dione has been made by M. Rapaport (09.100.003), and T. Nakamura (12.100.209), and a study of Japetus' motion, using new observations made at the Royal Greenwich Observatory, by A. T. Sinclair (12.100.214). The orbit of Uranus' satellite Miranda has been studied by R. J. Greenberg and E. A. Whitaker (10.101.011 and .018 and 11.101.003). R. J. Greenberg has studied the Laplacian-type relation amongst Uranus' satellites $(M N 173,121,1975)$. The origin of Uranus' satellite system has been considered by S. F. Singer (Icarus 25, 484, 1975). The orbit of Neptune's smaller satellite Nereid has been studied by L. E. Rose (11.101.007), and $\mathrm{F}$. Miguard $(A A 43,359,1975)$. Tidal friction and other mechanisms for the formation of the commensurabilities within the satellite systems have been studied by G. Colombo, F. A. Franklin and I. I. Shapiro (11.100.203), S. F. Dermott (12.099.227), R. J. Greenberg (09.091.042, IAU Symp. 62, 71, 1974, and $M N$ 170, 295, 1975), and A. T. Sinclair (11.100.201). Possible tidal influence on Uranus' system has been considered by E. L. Ruskol (10.101.004), possible capture of Jupiter's outer satellites by D. R. Davis (12.099.233), and the consequences of the principle of 'least interaction action' on the Laplace relation in Jupiter's system by M. W. Ovenden, T. Feagin, and O. Graf (11.099.202).

Considering now the dynamics of minor planets, reviews have been given by G. A. Chebotarev (12.042.088), N. S. Samojlova-Yakhomtova (10.098.018, and 12.098.001), and P. Herget (11.098.009). The masses of the two minor planets Ceres and Pallas have been estimated by J. Schubart (11.098.001 and $A A 39,147,1975)$. The mass of Saturn has been estimated from the motion of the Trojan minor planets by H. Scholl (09.100.019). The potentialities of radar ranging in minor planet orbit determinations have been studied by L. F. Bragar (09.098.068), and the latter with $\mathrm{Yu}$. V. Batrakov (09.098.010). Schemes of differential correction have been studied and used in minor planet orbit investigations by L. E. Sikhopluyeva (09.098.066 and $.067,10.098 .020,12.098 .066$ and .077$)$. Improved orbital elements for a large number of minor planets have been derived by F. B. Khanina (12.098.002 and .005), M. A. Dirikis (BITA $14,12,1975)$, and V. I. Orelskaya (BITA 14, 95, 1975). There has been a great deal of work on orbits strongly influenced by near-commensurability of period, including that of G. A. Chebotarev (10.098.023), the latter with N. A. Belyaev and R. P. Eremenko (12.098.031) on Trojan type orbits, that of P. K. Seidelmann, P. M. Janiczek, and G. H. Kaplan (10.042.050), H. Scholl and R. Giffen (12.098.032), O. A. Belousova, F. I. Isaeva and G. F. Sultanov (Tzirk. Shemaha Astrophys. Obs. 36, 3, 1974, and Dokl. Akad. Nauk Azerb. SSR 7, 1975), and R. Greenberg and F. Franklin $(M N 173,1,1975)$ on the Hecuba group $(2: 1$ resonance with Jupiter), that of H. Scholl and C. Froeschle (12.098.008) on the 3:1 commensurability, the same two authors 
on the $2: 1,5: 2$, and $7: 3$ cases $(A A 42,457,1975)$, that of E. A. Grebenikov, S. V. Mironov and V. A. Prihodko (10.098.034) on the Minerva group (5:2 resonance), that of G. A. Chebotarov, N. A. Belyaev and M. Yu. Shmakova (12.042.001) on both the $3: 1$ and 5:2 cases, and that of R. Giffen (09.098.002) on the Hecuba and Hilda cases. W. H. Ip and R. Mehra (09.098.006) have studied some cases of resonance with the Earth, while J. G. Williams and G. W. Wetherill (10.098.005), and R. L. Duncombe, P. M. Janiczek and P. K. Seidelmann (11.098.005) studied (1685) Toro, which can experience resonance with both the Earth and Venus. E. Z. Hotimskaya and M. S. Petrovskaya $(C M 11,265,1975)$ have developed a method for group calculation of perturbations. Y. Kozai and M. Yuasa (12.098.033), by study of higher-order secular terms, give estimates of the ages of some of the Hirayama families. Amongst work relating to the very long term history of the minor planet system, we note that of D. C. McAdoo and J. A. Burns (09.098.001), M. Lecar and F. A. Franklin (10.098.069), J. V. Breakwell (11.098.007), L. Dubkowski and S. Kasperczuk (11.098.020 and .021), S. Gaska (11.098.019 and 12.098.030). W. H. Ip (11.098.004), Gp. Horedt (12.042.049), G. F. Sultanov (Akad. Nauk Azerb. SSR Izv. 5, 89, 1974), H. Alfvén, W. H. Ip and M. D. Burkenroad (12.098.009), and T. A. Heppenheimer ( $A J$ 80, 465, 1975, and Icarus 26, 367, 1975). The tendency of recent work has been towards the view that the Kirkwood gaps arose from the effect of collisions amongst minor planets, though the last-named author challenges the basis of this mechanism.

A review of work on comets has been made by B. G. Marsden (11.102.010), who has continued his studies of non-gravitational forces, with Z. Sekanina and D. K. Yeomans, considering Encke's comet (09.102.008 and 11.103.102), has discussed the comet of the Perseid meteors (10.103.107), and the 'original' orbits of long-period comets, with Z. Sekanina $(A J 78,1118$, 1973). Non-gravitational forces have also been considered for comets 1939 III and $1925 \mathrm{VI}$ by K. A. Steins, L. V. Divina and I. A. Revina (Ucen. zap. Latv. gos. Univ. 175, 38, 1973). T. Kiang (09.103.103) has studied long-period effects in the motion of Halley's comet. L. M. Belous has studied the long-term evolution of the orbit of comet Borelly (1905II) (12.103.100), of comet Brorsen-Metcalf (1847V, identified with 1919III) (11.103.117 and 12.103.102), and comet Westphal (1852IV, identified with 1913VI) $(12.103 .101$, and $T K A O$ 40, 69, 1974). N. A. Bokhan and Yu. A. Chernetenko have carried out numerical integrations for the orbit of comet Encke-Backlund (1786I) (11.103.102 and 12.103.110). N. A. Belyaev has studied the long-term evolution of the orbit of comet Churyumov-Gherasimenko (1969 IV), which makes thirteen close approaches to Jupiter between 1660 and 2060 (10.103.131), of comet Neujmin 2 (1916 II) (TKAO 39, 102, 1973), comets Gunn (1969 II) and Kojima (1970 XII) with E. A. Reznikov (TKAO 39, 110, 1973), and comet Schwassmann-Wachmann (1930 VI) with S. D. Shaporev (12.103.109). L. Ya. Ananyeva and E. D. Kondratyeva (TKAO 40, 64, 1974) have studied the orbit of comet Pons-Winnecke (1819 III), L. A. Markelov (TKAO 40, $79,1974)$ has studied comet Everhart (1974 XII), and I. Evdekimov and Yu. Evdekimov (TKAO 40, 47, 1974) studied comet Giacobini-Zinner (1900 III). M. P. Imnadze (12.042.043) has developed a method for the refinement of orbital elements at one apparition. S. Vaghi (09.102.005 and .017) has considered the origin of Jupiter's family of comets, and E. Everhart (09.102.012) has reviewed various ideas on comet origins.

Turning to theories of artificial satellite motion, J. Henrard (12.052.036) shows how virtual singularities may be avoided by the use of Lie transforms, though D. G. Saari (11.052.004) shows that regularisation in the usual sense cannot be applied. Studies of zonal harmonic perturbations have been continued by $\mathrm{H}$. W. Milnes (09.042.016), who gives a closed-form solution for motion in the equatorial plane, N. V. Emelyanov (10.042.004), G. A. Krasinskij (10.052.027). L. P. Nasanova (10.042.003), who gives third-order secular perturbations, using an intermediary of the type given by Aksenov and Vinti, R. Ramnath (10.052.003), and T. A. Agekyan (11.042.003). A. Kamel and R. Tibbits (10.052.002) have studied optimal initial positions of the node for minimum excursion in latitude. B. Garfinkel (10.052.001) gave a global solution of the critical inclination problem, and A. H. Jupp (CM 11, 361, 1975) studied the extension of this problem with the inclusion of the harmonics $J_{3}, J_{4}$, and $J_{5}$, finding possible 'double libration' for small ranges of the parameters. Lunar and solar perturbations were studied by G. E. O. Giacaglia (11.052.007), E. I. Tomoshkova and K. V. Kholshovnikov 
(11.042.068), M. L. Lidov (12.042.033), R. H. Estes (12.052.024), and by V. Sokolov (09.052.044), who considered the case of luni-solar resonance. Atmospheric drag was the subject of study by E. I. Aksenov and B. N. Noskov (09.052.022), and the latter (12.052.002), A. M. Fominov (10.052.080, and 12.052.001), and by J. S. Watson, G. D. Mistretta and N. L. Bonavita ( $C M 11,145,1975)$ who propose an analytical method for computing drag perturbations in Vinti's theory. Perturbations due to radiation pressure have been studied by E. N. Polyakhova (09.052.038), N. V. Motorina (Akad. Nauk Moldav. SSR Izv. 3, 33, 1973), S. N. Vashkcvỳak (12.052.039 and Vestn. Moskov gos. Univ., phys. abstr. 15, 584, 1974), L. K. Grinevitskaya and E. N. Polyakhova (10.052.017 and .040), who considered the motion of a vehicle with a solar sail, and Yu. N. Isayev $(10.052 .012, .013$, and .014$)$, who considered also zonal harmonics and luni-solar perturbations on a balloon satellite. Theories for a geostationary satellite have been given by W. Flury (09.052.012), M. A. Vaçhkovyak and M. L. Lidov (09.052.026), A. Kamel, D. Ekman and R. Tibbits (10.052.006), Sh. T. Khabibullin (TKAO 40, 3, 1975), and S. G. Zhuravlev (10.052.031 and 032). The use of a non-inertial reference frame has been studied by G. Balmino (12.052.035), N. A. Beleayev (BITA 13, 477, 74), Y. Kozai and H. Kinoshita (09.052.011 and 10.042.006), K. Lambeck (09.052.002), and V. P. Semenko (12.042.066). G. Janin has studied accurate methods for orbits of high eccentricity (12.052.037), A. Pavlov and V. A. Sapa studied perturbations by the magnetic field on an orbiting dipole (12.052.005), and P. Musen and T. Felsentreger the determination of longperiod tidal perturbations (09.052.003). Questions of orbit determination and improvement have been considered by D. W. Curkendall andC. T. Leondes (11.052.001), K. V. Kholshevnikov (09.042.056), S. A. T. Long (CM 12, 225, 1975), andV. A. Tamarov (10.052.034).

Determinations of the coefficients of the harmonics of the Earth's field have been made by E. M. Gaposhkin (12.081.037), D. G. King-Hele and G. E. Cook (11.081.021), and C. A. Wagner (09.081.024), and the representation of the field considered by A. Marussi (11.081.023). Particular coefficients of high-order harmonics have been determined from satellites passing through the fifteenth-order resonance by D. G. King-Hele and D. M. C. Walker (09.054.014), and, with R. H. Gooding, (11.081.019 and Planetary Space Sci. 23, 1239, 1975), D. G. King-Hele (11.081.001), the latter with A. N. Winterbottom (12.081.007), and D. M. C. Walker (11.082.005, and .006, and Planetary Space Sci. 23, 565, 1975). A relation between the eleventh-order harmonics has been derived from the eleventh-order resonance of Vanguard 3 by C. A. Wagner (12.081.011). Tidal parameters have been estimated from Geos 1 and Geos 2 by B. C. Douglas, J. G. Marsh and N. E. Mullins (09.054.002), B. C. Douglas, S. M. Klosko, J. G. Marsh and R. G. Williamson (10.052.008), and K. Lambeck (12.052.009).

The theory of the rotation and libration of artificial satellites has been studied by V. V. Beletskij (09.042.037), V. G. Demin (Kosmich. Issled. 13, 158, 1975), V. V. Dobronravov (12.042.067), J. B. Eades and J. W. Drewry (09.042.001), P. J. Hamill and L. Blitzer (11.042.015), I. S. Kozlov $(A Z$ 52, 154, 1974), D. C. McAdoo and J. A. Burns (11.042.007), A. A. Nemo (09.042.023), R. Pringle (09.042.045), W. Rumyantsev (12.052.040) and S. I. Vereshihagina (12.042.089). Periodic motions were studied by V. G. Demin and F. I. Kiselev (11.042.004), V. J. Modi and K. C. Pande (CM 11, 195, 1975), and N. X. Vinh (10.042.060). The stability of such motions has been studied by V. A. Grobov and I. I. Kantemin (12.042.026), S. G. Zhuravlev (10.042.013 and 12.042.064), and V. A. Zlatoustov and A. P. Markeev (09.042.002). Also F. Boigey (09.042.052) and J. L. Junkins, D. Jacobson, and J. N. Blanton (09.042.039) deal with free motion of a rigid body in general, as do H. S. Morton, J. L. Junkins and J. N. Blanton (12.042.057), who give a solution in terms of Euler parameters. Motion of a rigid body in the restricted three body problem has been studied by W. J. Robinson (10.042.034 and $.085,12.042 .005)$, and the motion of three rigid bodies by G. N. Duboshin (11.042.010 and .057, and 12.042.045), V. T. Kondurar (12.042.060), M. Pascal (09.042.031 and $C M 12,337,1975)$, and V. V. Vidyakin (11.042.006, 12.042.046, and $A Z 52,152,1975)$. Effects of magnetic fields on satellite rotations have been studied by V. V. Beletskij and A. B. Novogrebelskij (09.052.007), and I. Stellmacher (10.052.003). Attitude control is treated by P. C. Hughes (11.052.002), and by R. Rangarajan and R. C. Flanagan (CM 12, 231, 1975).

The motion of a satellite of the Moon is the subject of studies by A. J. Ferrari (09.094.006), the latter with W. G. Heffron (10.052.005), R. F. Forga $(10.053 .010$ and $A A 44,25,1975)$, 
D. S. Ingram and B. Tapley (11.094.005), G. G. Koman $(11.052 .034,12.053 .022$, and $A Z 52$, 207,1975 ), and E. I. Timoshkova and K. V. Kholshevnikov (11.042.068). Questions relating to space trajectory variation, tracking, and orbit improvement, have been considered by $\mathrm{K}$. Aksnes (10.052.004), S. M. Altman (CM 11, 405, 1975), J. V. Breakwell, A. A. Kamel and P. J. Patner (12.052.025), and W. Kundt (09.052.021). The mass of Mars, and many of the coefficients of its gravity harmonics, have been derived from analysis of the orbit of Mariner 9 by J. L. Lorell and I. I. Shapiro (10.097.029), G. H. Born (12.097.073), and J. F. Jordan and J. Lorell (Icarus $25,146,1975$ ). The mass of Jupiter, the harmonic coefficients $J_{2}$ and $J_{4}$, and the masses of the four Galilean satellites have been determined from Pioneer 10 Doppler data by J. D. Anderson, G. W. Null and S. K. Wong (11.099.204, and 12.099.063).

In the gravitational problem of $n$-bodies, central, and other, configurations with special symmetries and collineations have been studied by P. Havas (09.042.018), J. S. Griffith (09.042.017), and E. M. Nezhinskyj (12.042.044), singularities and collisions by D. G. Saari (09.066.131, Dynamical Systems 479, 1973, and Trans. Amer. Math. Soc. 181, 351, 1973), and J. Waldvogel (10.042.066). On methods of regularisation of such singularities, D. C. Heggie $(12.042 .020)$, V. F. Myachin $(12.042 .002)$, V. Szebehely $(12.042 .017)$, and K. Zare (12.042.019), criteria for escape and capture were studied by A. S. Baranov (CM 11, 517, 1975), J. S. Griffith (12.042.038), and R. B. Patnaik (CM 12, 383, 1975), and other analytical questions by D. Greenspan (10.042.098 and .099), D. C. Heggie (12.151.011), L. Losco (10.042.010), and D. G. Saari (10.042.014, 12.042.071, and 12.160.014). An extensive discussion, both numerical and analytical, of the evolution of binaries has been made by $D$. C. Heggie (MN 173, 729, 1975). Numerical studies are reported by V. Szebehely (12.117.041 and 12.151.009), and, in the four-body problem, by R. S. Harrington (11.042.058), and by J. I. Katz (Icarus 25, 356, 1975).

In the general problem of three bodies, classification of solutions by use of the integrals was undertaken by B. Elmabsout $(09.042 .012,11.042 .029$ and 12.042.011), by D. G. Saari (S.I.A.M. J. Appl. Math. 26, 806, 1975), the latter with C. Marchal (CM 12, 115, 1975), generalising the concept of 'Hill's surfaces', and by C. C. Tung (12.042.011). The averaged problem has been studied by G. A. Krasinskij (12.042.032), and the irreducibility of the problem by Staekel's method by E. A. Grebenikov and M. N. Kyosa (J. Appl. Math. Mech. 33, $2,1974)$. Conditions for escape or retention were studied by J. S. Griffith and R. D. North (11.042.009), by C. Marchal (11.042.040), by D. G. Saari (11.042.031), and by J. Yoshida (12.042.012). A formulation of the equations for relative motion has been proposed by R. Broucke and H. Lass (10.042.011), and a method of regularisation by $S$. J. Aarseth and K. Zare (12.042.098). Resonances in the variational frequencies for the Lagrangian equilateral triangle solutions have been studied by A. L. Kunitsyn (11.042.059), and collision solutions in rectilinear motion with a preponderant mass by Mme. Irigoyen (11.042.061). Surveys of results obtained in numerical explorations have been made by V. Szebehely $(10.042 .070$, and .111, and 11.042.038 and .078), and by M. J. Valtonen (12.042.040 and Mem. Roy. Astron. Soc. 80, $61,76,1975)$. Triple close approaches have been studied by T. A. Agekyan and A. I. Martynova (09.042.055), V. Szebehely $(10.042 .015)$ and J. Waldvogel $(C M 11,429,1975)$, and capture and escape by V. Szebehely (09.117.002) and Gp. Horedt (11.042.077). Calculations of periodic solutions have been made by V. Szebehely and T. Feagin (10.042.012), generated from solutions of the elliptic restricted problem, by M. Henon (12.042.061), giving one-parameter families for a given mass-ratio, by R. Broucke and D. Boggs $(C M 11,13,19,75)$, by J. D. Hadjidemetriou $(11.042 .074, C M 12,155,175$, and 255,1975$)$, generated by continuation from periodic solutions of the circular restricted problem, and by G. Bozis and Th. Christides ( $C M$ 12, 277, 1975), who consider periodic motion around the collinear Lagrangian solutions.

Considering now analytical developments in the circular restricted problem of three bodies, L. M. Perko (S.I.A.M. J. Appl. Math. 27, 200, 1974), the latter with J. V. Breakwell (11.042.056), and P. Guillaume (CM 11, 449, 1975) have studied the use of matching techniques in the construction of asymptotic expressions, L. A. Moskovkina (09.042.054) has developed a general algorithm for the Hill-Brown method applied to the problem, P. Sconzo $(10.042 .035)$ has generalised the ' $f$ and $g$ ' series for the problem, M. L. Lidov and S. L. Ziglin (11.042.030) have studied the secular terms, finding a 'critical inclination', W. Wiesel 
(12.042.056) and H. Ruegger (dissrtn., E.T.H., Zurich, 1973) introduced regularised canonical parameters, E. A. Grebenikov and M. N. Kiosa (11.042.033) discussed the analytical structure of the partial derivatives of the Hamiltonian, and M. B. Faintlich (10.042.031) gave perspective drawings of the zero-velocity surfaces in the three-dimensional problem. V. G. Demin and A. L. Kunitsyn (10.042.109) have studied the limiting case of the two fixed centres problem, and I. S. Kozlov (10.042.054 and 12.042.081) has developed periodic solutions in the restricted problem by continuation from solutions of the two fixed centres problem. F. Schmeidler (10.042.044) studied periodic solutions by analytical continuation, A. Bruno (dissrtn., Moscow, 1973) studied such solutions in the limiting case of zero mass ratio, and J. Henrard (09.042.042) proved a conjecture of E. Stromgren on the conditions for a doubly-asymptotic solution to be the limit of a family of periodic solutions. Turning to numerical exploration of the solutions of the problem, studies of homoclinic points and Henon stability have been made by L. D. Mullins and J. H. Bartlett (09.042.041), J. M. A. Danby (10.042.029), and W. H. Jefferys (11.042.080), while V. V. Markellos (11.042.039, 12.042.008, and $C M 12,215$, 75) explored bifurcations of families of periodic solutions for the Jupiter mass ratio, finding the regions of stability and of instability. M. K. Zikides (10.042.002), the latter with G. Contopoulos (11.042.019), D. Benest (11.042.041), and V. V. Markellos, W. Black and P. E. Moran (11.042.062, and Astrophys. Space Sci. 33, L29, 1975) studied the evolution of families of orbits with change of mass ratio, the latter collaborators also studying periodic solutions corresponding to the Jupiter satellite case (Astrophys. Space Sci. 33, 385, 1975). P. G. Kazantzis and C. L. Goudas (12.042.048), and C. G. Zagouras and C. L. Goudas (12.042.052 and .079) developed a 'predictor-corrector' method for tracing families of periodic solutions. P. Guillaume, in a beautiful series of papers, studied symmetric periodic solutions of the second species, using many techniques $(10.042 .020$ and $.077,12.042 .076, A A 37,209,1974$, and $C M$ $11,213,1975)$. Collision solutions were studied by V.S. Károlyov (12.042.084). M. Hénon $(10.042 .028$ and .057 , and 11.042 .002$)$ studied the stability of plane periodic solutions to out-of-plane disturbances, and Y. Kozai andH. Kinoshita (09.042.007) studied solutions of the third sort associated with the commensurabilities $2: 1,3: 2$, and 6:1. C. N. Fragakis (10.042.008 and .009) used a phase plane analysis method, and found a new family of asymmetric periodic solutions associated with the 3:1 commensurability. Trojan type librations, and 'horse-shoe' orbits were studied by E. Everhart (09.042.032), the latter with M. Hollabaugh (10.042.088), Gp. Horedt (10.042.007), P. R. Weissmann and G. Wetherill $(11.042 .043)$, T. K. Shinkarik (12.042.003), J. Koels (CM 12, 327, 1975), and J. I. Katz (Icarus 25, 356, 1975). Solutions associated with the Lagrange collinear positions were studied by $F$. Nahon (10.042.016), I. V. Kurcheeva (10.042.039), and G. I. Shirmin (11.042.032, and $C M 11,483,1975)$. G. I. Tjivanidis and V. V. Markellos (Astrophys. Space Sci. 35, 151, 1975) studied 'boomerang' orbits. The relativisitic restricted problem of three bodies has been studied by $\mathrm{K}$. Dosybekov (12.042.009 and .010), and V. Singkh (12.042.082), R. K. Sharma (12.042.072), and the latter with P. V. Subba Rao ( $A A 43,381,1975$, and $C M 12,189,1975)$, have studied the motion when the primaries are oblate spheroids, and Gp. Horedt $(12.042 .059)$ has studied the stability of the Lagrange equilateral triangle positions when the primaries have variable mass.

Turning now to the elliptic restricted problem, formal series expansions for the collinear case were given by J. Waldvogel (10.042.019), and expansions in powers of the eccentricity of the relative orbit of the two finite bodies were considered by B. Erdi (11.042.065). Studies of motion near the equilateral triangle configurations were continued by E. Rabe (10.042.017 and $.076)$, who carried the analysis of stability to the fourth order, by J. Tschauer (10.042.018 and 11.042.055), who gave boundaries of stability in $\left(e^{\prime}, m^{\prime}\right)$-space, as did A. P. Markeev (10.042.033 and 11.042.070), by R. K. Choudhry (10.042.097), by M. Calvo (12.042.090), by $\mathrm{T}$. Heppenheimer (09.042.008), who studied out-of-plane motions, by V. Matas (10.042.036, 11.042.046 and .047, and Bull. Astron. Inst. Czech. 26, 34, 1975), who gave also results relating to the collinear configurations, as did R. W. Farquhar and A. A. Kamel (09.042.043). The existence of periodic solutions of the second kind was shown for a a particular case by A. Sergysels-Lamy (CM 11, 43, 1975). V. Matas (11.042.045 and Bull. Astron. Inst. Czech. 26, $30,1975)$ has studied periodic solutions with additional forces present.

The following books have been published during the three-year period under review: 
Balk, M. B., Demin, V. G. and Kunitsyn, A. L.: Collection of Problems of Celestial Mechanics and Astrodynamics. Manual for College Students. (in Russian) Nauka, Moskva, 1973.

Gavryuk, M.I.: Astronavigational Determination (in Russian), Transport, Moskva, 1973.

Gribbin, J. and Plagemann, S.: The Jupiter Effect: the Planets as Triggers of Devastating Earthquakes, Walker, New York, 1974.

Hagihara, Y.: Celestial Mechanics vol. IV, Japan Soc. for Promotion of Sci., Tokyo, 1975.

Morando, B.: Mouvement d'un satellite artificiel de la Terre, Gordon and Breach, Paris.

Moser, J.: Stable and Random Motions in Dynamical Systems (with Special Emphasis on Celestial Mechanics), Princeton Univ. Press, 1973.

Moulton, F. R.: An Introduction to Celestial Mechanics, reprinted by Dover, New York, 1972.

Norville, W.: Celestial Navigation Step-by-Step, Internat: Marine Publ. Co., Camden, Maine, 1973.

Pilcher F. and Meeus, J.: Tables of Minor Planets, private publ., 1973.

Stumpff, K. and Meffroy J.: Himmelsmechanik, Vol. III, Allgemeine Storungen, V.E.B. Deutscher Verlage der Wissenschaften, Berlin, 1974.

Whitrow, G. J.: What is Time?, Thames and Hudson, London, 1973.

Zhakharov, V. D.: Gravitational Waves in Einstein's Theory of Gravitation (transl. into English), John Wiley and Sons, Chichester, 1973. 